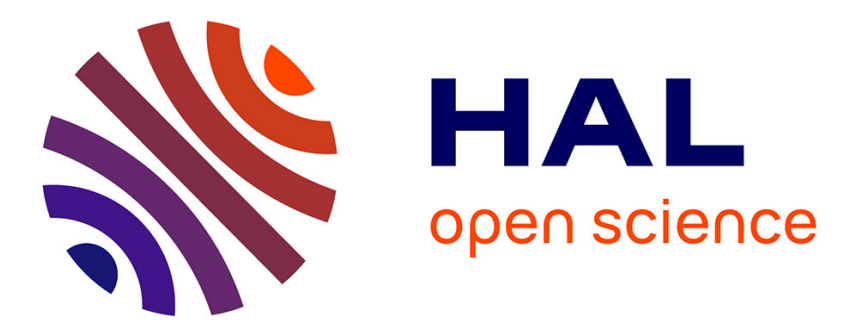

\title{
Modular regulation analysis of integrative effects of hypoxia on the energetics of contracting skeletal muscle in vivo
}

Christophe Beuste, Sylvain Miraux, Véronique Deschodt-Arsac, Eric

Thiaudiere, Jean-Michel Franconi, Philippe H Diolez, Laurent M Arsac

\section{To cite this version:}

Christophe Beuste, Sylvain Miraux, Véronique Deschodt-Arsac, Eric Thiaudiere, Jean-Michel Franconi, et al.. Modular regulation analysis of integrative effects of hypoxia on the energetics of contracting skeletal muscle in vivo. Biochemical Journal, 2009, 420 (1), pp.67-72. 10.1042/BJ20082385 . hal-00479138

\section{HAL Id: hal-00479138 \\ https://hal.science/hal-00479138}

Submitted on 30 Apr 2010

HAL is a multi-disciplinary open access archive for the deposit and dissemination of scientific research documents, whether they are published or not. The documents may come from teaching and research institutions in France or abroad, or from public or private research centers.
L'archive ouverte pluridisciplinaire HAL, est destinée au dépôt et à la diffusion de documents scientifiques de niveau recherche, publiés ou non, émanant des établissements d'enseignement et de recherche français ou étrangers, des laboratoires publics ou privés. 


\section{Modular regulation analysis of integrative effects of hypoxia on the energetics of contracting skeletal muscle in vivo}

Christophe BEUSTE, Sylvain MIRAUX, Véronique J. DESCHODT-ARSAC, Eric THIAUDIERE, Jean-Michel FRANCONI, Philippe DIOLEZ, Laurent M. ARSAC.

Résonance Magnétique des Systèmes Biologiques, UMR 5536 Centre National de la Recherche Scientifique (CNRS) - Université Bordeaux 2, Bordeaux, France.

SHORT TITLE : hypoxic regulation of muscle ATP turnover: top-down analysis 


\section{Synopsis}

In exercising muscle, acute reduction in ambient oxygen impairs muscle contraction due to effects of hypoxia on mitochondrial ATP supply. The less marked impairment reported after long-term exposure to hypoxia points to changes in regulation of the energetic system of contraction in hypoxic conditioned animals. This energetic system was conceptually defined here as two modules, ATP/PCr-producer and ATP/PCr-consumer connected by energetic intermediates. Modular control analysis (MoCA) that combines top-down control analysis with non-invasive ${ }^{31} \mathrm{P}-\mathrm{NMR}$ spectroscopy was used to describe in vivo the effects of hypoxia and adaptation to hypoxia on each module.

Modulations of steady levels of ATP turnover (indirectly assessed as force output) and muscle PCr were obtained in hypoxic conditioned $(\mathrm{HC})$ rats $\left(6\right.$ weeks at $\left.10.5 \% \mathrm{O}_{2}\right) v s$. normoxic $(\mathrm{N})$ rats. Modular control and regulation analyses quantified the elasticity to $\mathrm{PCr}$ of each module in $\mathrm{N}$ and $\mathrm{HC}$ rats as well as the direct effect of acute hypoxia on the PCr/ATP-producer module.

Similar elasticities in N and HC rats indicate the absence of response to long-term hypoxia in internal regulations of the ATP supply and demand pathways. The less marked impairment of contraction by acute hypoxia in $\mathrm{HC}$ rats $(-9 \pm 6 \% v s .-17 \pm 14 \%$ in $\mathrm{N}$ rats, $P<0.05)$ was therefore fully explained by a lower direct effect $(\mathrm{HC}-31 \pm 13 \%$ vs. $\mathrm{N}-44 \pm 23 \%, P<0.05)$ of acute hypoxia on mitochondrial ATP supply. This points up a positive adaptation to chronic hypoxia.

MoCA in vivo may provide powerful tools to find out improved function (alternatively dysfunction) at system level in conditioned animals.

Keywords. Metabolic control analysis; ${ }^{31}$ P-MR Spectroscopy; ATP turnover; hypoxic conditioning; oxidative phosphorylation 


\section{Introduction}

As an effective oxygen sink, mitochondria house the ultimate step of the fate of oxygen pathway from air through the cardiovascular and pulmonary systems. Oxygen is used for substrate oxidation by mitochondria and is therefore involved in the main mechanism responsible for energy supply in moderately exercising skeletal muscle. An intriguing question for physiologists over the last three decades has been the influence of acute hypoxia over muscle contraction in an integrated context. Accordingly, the effect of breathing gas mixtures with varied fractions of inspired oxygen on muscle respiration has been extensively studied. Acute and severe reduction in oxygen delivery typically results in a wide range of metabolic disturbance, e.g. decrease in the steady level of phosphocreatine ( $\mathrm{PCr})$ [1] and alteration in other energetic intermediates (ATP, Pi, and ADP) [2], ultimately culminating in a total loss of contractile function [3, 4]. A less marked perturbation by acute hypoxia of the bioenergetic system of contraction in skeletal muscle is generally reported in humans as well as in rodents after exposure to hypoxia for several weeks as well as in humans indigenous to high altitudes $[5,6]$. To date yet, the hypoxia-induced responses of integrative regulatory features of muscle energetics have not been systematically studied.

Recent successful analyses suggest that an improved understanding of the integrative regulation by $\mathrm{O}_{2}$ could be gained from a modular approach of the energy metabolism of contracting muscle. In such integrative approaches, the energy metabolism of intact contracting muscle (either heart or skeletal muscle) can be conceptually divided into two modules: ATP/PCr-producer and ATP/PCr-consumer [7-10]. Modules communicate only via a group of energetic intermediates (ADP, ATP, $\mathrm{Pi}, \mathrm{Cr}$ and $\mathrm{PCr}$ ) whose concentrations are connected to each other and to the phosphorylation potential. Since muscle PCr dynamics can be detected non-invasively by 31P-MR spectroscopy with high signal/noise ratio, $\mathrm{PCr}$ concentration ([PCr]) is systematically the studied variable in the above experimental works. The reactivity of each module to PCr is quantified by the elasticity coefficient (or elasticity). Since the whole set of elasticities determine internal regulation in a metabolic pathway [1115] as well as in modular systems [7-10, 14, 16, 17], these parameters may be used to objective the regulation of ATP turnover or so-called 'energy demand-supply coupling' [2, 
$5,6,18]$ in contracting muscle of hypoxic animals. Additionally, once modular elasticities are known, the regulatory role of any effector on the energetic system can be quantified [9, 12]. So, the overall regulation analysis would likely make it possible to find out the origin of the less marked perturbation by $\mathrm{O}_{2}$ of the energetic system of contraction after hypoxic conditioning.

In the present study, ${ }^{31} \mathrm{P}-\mathrm{MR}$ spectroscopy was used to quantify in vivo the elasticity to $\mathrm{PCr}$ of ATP/PCr-producer and ATP/PCr-consumer modules in normoxic rats (N) vs. hypoxic conditioned rats $(\mathrm{HC})$ exposed for 6 weeks to $10.5 \% \mathrm{O}_{2}$. The determination of elasticity values then served for the determination of control exerted by each module on ATP turnover and contraction, as quantified by flux-control coefficients. At last, thanks to the determination of control distribution, the direct effect of $\mathrm{O}_{2}$ on the producer module was quantified in $\mathrm{N}$ and $\mathrm{HC}$ rats to uncover regulatory features of acute hypoxia on the energetic system.

\section{Experimental procedures}

Animal preparation. Female Wistar rats weighting 280 to $340 \mathrm{~g}$ were used for these experiments according to the guidelines of the National Research council's Guide for the Care and Use of Laboratory Animals. All animals were housed four to cage in an environmentally controlled facility $\left(12 \mathrm{~h} / 12 \mathrm{~h}\right.$ light-dark cycle, $\left.22^{\circ} \mathrm{C}\right)$ and received food and water ad libitum. They were separated in two groups: normoxic $(\mathrm{N}) v s$. chronically hypoxic $(\mathrm{CH})$, each group housed in specific compartments.

In the hypoxic compartment, long-term reduction in the fraction of inspired oxygen $\left(\mathrm{FiO}_{2}\right)$ to $10.5 \%$, as achieved by mixing air with nitrogen, was used to simulate chronic hypoxia during six weeks. Circulating the gas mixture through a water volume into a mixing chamber preserved humidity. $\mathrm{F}_{\mathrm{i}} \mathrm{O}_{2}$ was continuously monitored in the hypoxic compartment with an alarm oxygen analyser (GasAlert Extreme, BW technologies, Calgary, Canada).

For experiments, rats were carefully taken out of their cage then anesthetized in a plexiglass box flushed by air containing 3\% isoflurane. Throughout the experiment, anesthesia was 
maintained by continuous inhalation of a gas mixture containing $1.5 \%$ isoflurane delivered in a facemask.

Experimental set up. The experimental set up was described elsewhere [7]. Briefly, the anesthetized rat was placed in a supine position into a supraconducting magnet (4.7 Tesla, 47/50 Biospec Avance MR system Bruker, Ettlingen, Germany) so that the right segment of the lower limb was centred inside a $30 \mathrm{~mm}$ diameter ${ }^{1} \mathrm{H}$ Helmotz imaging coil tuned to the proton frequency $(200.3 \mathrm{MHz})$. A circular homebuilt transmit-receive ${ }^{31} \mathrm{P}$ probe $(18 \mathrm{~mm})$ was placed horizontally under the gastrocnemius and tuned to the phosphorus frequency $(81.1 \mathrm{MHz})$. Proton shimming was achieved locally in a $20 \times 20 \times 20 \mathrm{~mm}^{3}$ voxel by using the water proton resonance, with typical widths at half heights of $30-35 \mathrm{~Hz}$.

Before measurements in contracting muscles, a proton MR image was acquired axially by using the ${ }^{1} \mathrm{H}$ coil to verify muscle group position and to improve field homogeneity.

The plantar flexor muscles group was stimulated electrically (Compex 2 stimulator, Ecublens, Switzerland) directly with transcutaneous electrodes located at the knee and heel levels. For the study of moderate intensity of exercise, the current intensity was set at $6 \mathrm{~mA}$ in $\mathrm{N}$ rats as well as in $\mathrm{CH}$ rats.

A total of thirty ${ }^{31} \mathrm{P}$ spectra $\left(100 \mu \mathrm{s}\right.$ rectangular pulse, $60^{\circ}$ flip angle in the middle of the coil; 64 accumulations $2.8 \mathrm{~s}$ recovery time; $3.3 \mathrm{kHz}$ bandwidth; 1024 data points) were acquired in 180s blocks throughout the experimental protocol.

Two ${ }^{31} \mathrm{P}-\mathrm{MRS}$ spectra were acquired to assess the level of muscle phosphocreatine at rest. Then, during a warm-up period muscle stimulation was increased progressively and hypoxia was gradually initiated by $\mathrm{F}_{\mathrm{i}} \mathrm{O}_{2}$ reduction of inhaled gas $\left(21 \%\right.$ to $\left.9 \% \mathrm{O}_{2}\right)$. No measurements were taken into account for the subsequent analysis until contraction and spectra reached a steady state. Typically this transitory period lasted about $40 \mathrm{~min}$ (13 spectra).

Once the hypoxic $\left(9 \% \mathrm{O}_{2}\right)$ steady state was reached and maintained for $15 \mathrm{~min}$, a first switch to air for additional $15 \mathrm{~min}$ ( 5 spectra) followed by a second switch to a lower electrical stimulation (5 additional spectra) provided modulations around the studied steady state of $\mathrm{PCr}$ 
and muscle work rate. The elasticity coefficient of the producer and consumer blocks, fluxcontrol coefficients, and direct effect of $\mathrm{O}_{2}$ on producer (see below) were determined around this steady state.

System variables. The conceptual system of muscle energetics used here is composed of two modules, ATP/PCr-producer and ATP/PCr-consumer (fig 1). The analysis of the system requires the measurements of two variables: the intermediate and the flux through each module; the later is equal to the overall flux through the system under steady state conditions. The representative 'intermediate' in the system was $\mathrm{PCr}$ concentration, as assessed by ${ }^{31} \mathrm{P} \mathrm{MR}$ spectroscopy; muscle work rate represented the 'flux' as assessed by plantar flexion force exerted on a pedal [7].

Concentrations of metabolic intermediates were assessed from MR-spectroscopy spectra. MRS spectra were routinely deconvolved into Lorentzian lines (Igor Pro Wavemetrics, Lake Oswego, Oregon, USA). The phosphocreatine, $\mathrm{Pi}$, and $\beta$-ATP peak areas were calculated and were converted to concentrations assuming $[\mathrm{ATP}]=8.2 \mathrm{mM}$.

Contraction resulting from muscle electrical stimulation was measured with a homebuilt ergometer consisting in a foot pedal connected to a hydraulic piston. A hydraulic circuit filled with water connected the piston to a force transducer (ref. $\mathrm{N}^{\circ}$ MLT0699, AD Instruments powerLab Systems) that was placed outside the magnet. Changes in pressure induced by pedal stroke were recorded at $200 \mathrm{~Hz}$. The magnitude of strokes was computed as a function of time (Igor Pro, Wavemetrics, Oregon, USA) to provide the work rate (contraction) signal.

Modular Control Analysis. The experimental setup made it possible to quantify concomitant changes in steady levels of $\mathrm{PCr}$ and contraction during modulations around the studied steady state called 'reference'. Following the principles of Metabolic Control Analysis, the modulation by acute hypoxia of steady levels of $\mathrm{PCr}$ and contraction made it possible to determine two parameters critical for our understanding of integrative regulation 
of the modular system by acute hypoxia: (i) the consumer elasticity $\left(\varepsilon_{\mathrm{c}}\right)$ and (ii) the role of oxygen as an external effector of the producer module. The consumer elasticity was determined by relative change in contraction divided by relative change in $\mathrm{PCr}$, induced by acute hypoxia. The direct effect of acute hypoxia on the producer was determined by the relative change in contraction divided by the flux-control coefficient of the producer (see below). The modulation by electrical stimulation allowed the quantification of the producer elasticity $\left(\varepsilon_{\mathrm{p}}\right)$. The producer elasticity was determined by the relative change in contraction divided by the relative change in $\mathrm{PCr}$, induced by lowering electrical stimulation. Summation and connectivity theorems allowed the calculation of flux-control coefficients from the experimental elasticities. The flux-control coefficient of the producer $\left(\mathrm{C}_{\mathrm{p}}\right)$ was calculated as $\varepsilon_{\mathrm{c}} /\left(\varepsilon_{\mathrm{c}}-\varepsilon_{\mathrm{p}}\right)$; the flux control coefficient of the consumer $\left(\mathrm{C}_{\mathrm{c}}\right)$ equals: $\varepsilon_{\mathrm{p}} /\left(\varepsilon_{\mathrm{p}}-\varepsilon_{\mathrm{c}}\right)$.

Enzymatic analysis. Once all the non-invasive measurements were performed, the animal was sacrificed by the injection of a lethal dose of pentobarbital. A portion of the rat soleus muscle (about $80 \mathrm{mg}$ ) was rapidly excised with scissors and immediately cooled in ice-cold buffer consisting of $25 \mathrm{mM}$ Sucrose, $5 \mathrm{mM}$ MOPS and $0.2 \mathrm{mM}$ EDTA. Whole tissue homogenates were prepared in the same buffer by hand homogenisation using a Polytron (PT $1200 \mathrm{E}$; Kinematica $\mathrm{AG})$ at $4^{\circ} \mathrm{C}$. An aliquot of the homogenate $(150 \mu \mathrm{l})$ was used for measuring cytochrome c oxidase (Cox) activity, by following the decrease in absorbance at $550 \mathrm{~nm}$ in spectrometer (Cary $50 \mathrm{UV}$-Vis spectrophotometer; Varian Inc.) with chemically reduced horse heart cytochrome $\mathrm{c}$ as electron donor. The reaction mixture contained (in $\mathrm{mM}$ ): $100 \mathrm{KCl}, 40$ saccharose, $10 \mathrm{KH}_{2} \mathrm{PO}_{4}, 5 \mathrm{MgCl}_{2} / \mathrm{H}_{2} \mathrm{O}, 1$ EGTA and $0.1 \%$ (w/v) BSA (pH=7.2). The reaction was started by addition of reduced cytochrome c $(50 \mu \mathrm{M})$. Cox activity was expressed in $\mu \mathrm{mol}$. $\min ^{-1} \cdot \mathrm{g}^{-1}$ of protein.

Blood analysis. The hematocrit (Ht) was determined using blood collected from rat heart and centrifuged in micro capillary tube. 


\section{Results}

Hypoxic conditioned (HC) rats exposed to $10.5 \% \mathrm{O}_{2}$ during 6-weeks had higher hematocrit than control normoxic $(\mathrm{N})$ rats $(52 \pm 3 \%$ vs. $42 \pm 3 \%, \mathrm{P}<0.05)$, thus indicating improved oxygen transport capacity. HC rats had also lower Cox activity $\left(250 \pm 12\right.$ vs $343 \pm 27 \mu \mathrm{mol} \cdot \mathrm{min}^{-1} \cdot \mathrm{g}^{-1}$, $\mathrm{P}<0.05)$ possibly indicating a lower maximal oxidative capacity in the soleus muscle. Taken together, these results indicate that our rats exposed for 6 weeks to $10.5 \% \mathrm{O}_{2}$ were effectively conditioned.

The present elasticity analysis was based on simultaneous recordings of steady levels of contraction and PCr during a steady state exercise modulated by acute hypoxia $\left(9 \% \mathrm{O}_{2}\right)$ and slight decrease in electrical stimulation as illustrated in figure 2. Figure 3 provides a comparative overview of averaged changes in $\mathrm{PCr}$ and contraction due to modulations by $9 \% \mathrm{O}_{2}$ and lowering electrical stimulation in $\mathrm{N}$ rats and $\mathrm{HC}$ rats. Solid lines represent the effect of each modulation around the studied steady state called 'reference'. As quantified below by the elasticity analysis, figure 3 shows similar slopes (solid lines) in HC and $\mathrm{N}$ rats in response to each modulation. It also worth noting that acute hypoxia $\left(9 \% \mathrm{O}_{2}\right)$ induced a lower decrease in both $\mathrm{PCr}$ and contraction in $\mathrm{HC}$ rats when compared to $\mathrm{N}$ rats.

Averaged elasticities of the ATP/PCr-producer and ATP/PCr-consumer module in each population ( $\mathrm{HC} v s . \mathrm{N})$ are shown in table 1. One main finding of the present study was the absence of difference between elasticities in $\mathrm{HC}$ rats and in $\mathrm{N}$ rats.

Flux-control coefficients calculated from these experimental elasticities are reported in table 1. A very similar control distribution was quantified in $\mathrm{N}$ and $\mathrm{HC}$ rats. In the present study where moderate work intensity was stimulated, the control over ATP turnover and contraction was dominantly on the consumer $\left(\mathrm{C}_{\mathrm{c}}=65 \%\right)$. Yet, since the producer had substantial control over contraction $\left(\mathrm{C}_{\mathrm{p}}=35 \%\right)$, any external effector that can affect directly the producer activity is expected to alter contraction in some extent. Acute hypoxia $\left(9 \% \mathrm{O}_{2}\right)$ was just the effector whose direct effect on producer was quantified here. Since contraction was less inhibited by acute hypoxia in $\mathrm{HC}$ rats while the producer, which is the target of acute hypoxia, had similar control over contraction, a lower direct effect of acute hypoxia on 
producer must be concluded. Accordingly, the quantified direct effect of acute hypoxia on the ATP/PCr-producer module in $\mathrm{HC}$ rats was only $70 \%$ of the direct effect in $\mathrm{N}$ rats (table 1 ).

\section{Discussion}

The present study demonstrates that, in moderately contracting skeletal muscle, internal regulatory features of ATP turnover as determined here by elasticity coefficients in vivo are likely not affected by long-term exposure to $10.5 \% \mathrm{O}_{2}$. In agreement with previous works e.g. [6] we additionally observed a lower impact of acute hypoxia $\left(9 \% \mathrm{O}_{2}\right)$ on contraction in hypoxic conditioned animals, which can be viewed as a positive adaptation to chronic hypoxia. The application of regulation analysis indicates that in absence of change in internal regulation of ATP turnover, this adaptation in $\mathrm{HC}$ rats is fully explained by a lower direct effect of ambient hypoxia on the producer.

There is a growing appreciation of the concept that an improved understanding of complex regulatory processes could be gained from integrative determination of modular elasticity coefficients in intact organs. By conceptually defining heart energetics as a two-module system, ATP/PCr-producer and ATP/PCr-consumer, Diolez and colleagues [9] highlighted the routes taken by regulatory effects of calcium in beating heart. Korzeniewski and colleagues [10] incorporated elasticity coefficients in the Proportional Activation Approach and demonstrated so, that both ATP/PCr-producer and ATP/PCr-consumer modules are directly activated to a similar extent during physiological activation of the heart by adrenaline. As regards skeletal muscle, Jeneson and colleagues [17] and recently Arsac and colleagues [7] proposed theoretical and experimental approaches respectively for the assessment in vivo, of elasticity coefficients of ATP/PCr-producer and ATP/PCr-consumer modules. Although such methods are usually crude, they have been demonstrated to significantly strengthen the field of physiological regulations in skeletal muscle [16]. Since in the present study, the producer elasticity reached similar values in normoxic rats and hypoxic conditioned rats, we conclude that hypoxic conditioning has no effect on the reactivity of oxidative phosphorylation to energetic intermediates (ADP, ATP, Pi, PCr, $\mathrm{Cr}$ and the phosphorylation 
potential) during moderate exercise. In other words, the substrate feedback control [19, 20] of tissue respiration is likely unaltered by chronic hypoxia. Although our approach actually did not evaluate variations in all these key regulators but focused on $\mathrm{PCr}$ as representative, the degree to which PCr is broken down in our conditions fits well with changes of the energy charge of the cell $[2,18,21,22]$. Force output, as a surrogate of ATP turnover, was the other critical variable measured in the present approach. For reliable MCA, one should assume that contractile efficiency is not affected by hypoxia, incidentally fatigue due to prolonged work, or stimulation intensity. In humans cycling at submaximal intensities, no change in efficiency was observed at sea level, acute, and chronic altitude of 4300m [23]. As well, in electrically stimulated canine gastrocnemius under control, ischemic and hypoxemic conditions, there was clearly no effect of a lower $\mathrm{O}_{2}$ delivery on efficicency, as indicated by proportional decrease in muscle force output and $\mathrm{O}_{2}$ uptake [24]. In steady states prolonged for hours ( $>2 \mathrm{~h}$, personal results) we noted no decline in force output or in $\mathrm{PCr}$ concentration, even at the highest (sub-maximal) intensities used in these experiments. We concluded that electrical stimulation intensities generate non-fatiguing conditions and that efficiency therefore is unlikely to change with respect to time in our conditions. At last, an obvious candidate for a change in efficiency due to different stimulation intensities is a shift in the recruitment of oxidative and glycolytic fibers. Our previous $\mathrm{T}_{2}$-weighted images (based on ${ }^{1} \mathrm{H}$ MR imaging) indicated that fibers of the mixed gastrocnemius are recruited at whatever electrical intensity [7]. Furtherrmore, muscle pH during the periods of interest reached $6.97 \pm 0.04$ in $\mathrm{N}$ rats and $6.94 \pm 0.07$ in $\mathrm{HC}$ rats with no value below 6.87 , thus indicating that ATP synthesis is fully, or at least to a large extent, oxidative in every experiment.

Although the producer elasticity is a critical parameter that likely reveals kinetic or thermodynamic 'driving functions' for mitochondrial metabolism, the comprehensive understanding of ATP turnover regulation requires paying attention to the reactivity of ATP/PCr consumption as well. In the present study, we obtained similar consumer elasticities in $\mathrm{N}$ and $\mathrm{HC}$ rats. Therefore, similar $\varepsilon_{\mathrm{p}}$ as well as similar $\varepsilon_{\mathrm{c}}$ in both populations suggest that 
internal kinetic functions involved in the regulation of muscle ATP turnover in vivo are essentially not altered in hypoxic conditioned rats, as compared to normoxic rats.

The absence of changes in elasticity to the intermediates of ATP production and ATP consumption in hypoxic animals is not documented elsewhere in the literature. Our results in normoxic animals can yet be compared to experiments based on electrically stimulated skeletal muscle of animals in situ. Hogan and colleagues [2] obtained concomitant recordings of contraction and PCr levels in electrically stimulated canine gastrocnemius in situ at two levels of stimulation and under normal and low $\mathrm{O}_{2}$ conditions. So, the elasticity of conceptual producer and consumer modules in their normoxic animals can be calculated from concomitant changes in oxygen uptake and $[\mathrm{PCr}]$ in response to hypoxemia and electrical stimulation. The calculated values of -1.1 for the producer elasticity and 1.2 for the consumer elasticity are consistent with the present quantification in $\mathrm{N}$ rats (table 1). These similarities in elasticity coefficients obtained in intact muscle in previous studies [2, 7] as well as in the present study indicate that, although the proposed experimental methods might be crude, elasticity coefficients can be determined in intact muscle with fine consistency. Therefore, we exclude that $\mathrm{HC}$ rats might have altered elasticities undetected by our present approach and point definitively to the absence of alteration in internal regulatory features of ATP turnover and contraction in our hypoxic conditioned rats.

The theory of metabolic control analysis has shown that control over the flux through a given pathway can be distributed over many steps in the pathway and that the degree of control of any given step can be quantified by its flux control coefficient [15]. In the present study, the control over ATP turnover and contraction derived from elasticity values was effectively distributed between the two modules in each population (table 1). The quantification of fluxcontrol coefficients highlights similar $\mathrm{C}_{\mathrm{p}}$, amounting to $35 \%$ in $\mathrm{N}$ and $\mathrm{HC}$ rats. The critical consequence for our understanding of regulation by $\mathrm{O}_{2}$ of muscle energetics is that any direct effect of $\mathrm{O}_{2}$ on the producer activity will be transmitted to contraction with similar 'strength' (35\%) in $\mathrm{N}$ and $\mathrm{HC}$ rats. Therefore similar control strength over the flux by the producer module in $\mathrm{N}$ and $\mathrm{HC}$ rats has to be analyzed in concert with the overall effect of $9 \% \mathrm{O}_{2}$ on contraction (so-called global effect in the framework of regulation analysis). This global 
effect was only $-9 \pm 6 \%$ in $\mathrm{HC}$ rats but amounted to $-17 \pm 14 \%$ in $\mathrm{N}$ rats (figure 3 ). Since this difference in global effect cannot be explained by different control by the producer over contraction, it is fully explained by a lower direct effect of $9 \% \mathrm{O}_{2}$ on the producer module, amounting to $-31 \%$ in $\mathrm{HC}$ rats but $-44 \%$ in $\mathrm{N}$ rats $(P<0.05)$. It is therefore concluded that the target of hypoxic conditioning in the integrated energetic system of contraction in vivo is not the sensitivity of ATP/PCr producing processes to energetic intermediates - as reflected by elasticity coefficients - but the sensitivity of these processes to ambient $\mathrm{O}_{2}$. This is a critical advantage of regulation analysis to quantify direct effects of external effectors on a metabolic pathway. The $30 \%$ lower direct effect of $\mathrm{O}_{2}$ on mitochondrial ATP/PCr production quantified here in $\mathrm{HC}$ rats was obtained after 6 weeks at $10.5 \% \mathrm{O}_{2}$ in initially healthy rats. Different conditioning patterns may generate different strength of adaptation. Similarly, any initial pathology in the cardio-respiratory system of the animal may result in more or less severe change in the direct effect of acute hypoxia. Modular regulation analysis likely has the potential to quantify these particular integrated responses.

Our study was not designed to specifically identify the very mechanisms responsible for the lower sensitivity to ambient $\mathrm{O}_{2}$ of ATP/PCr production in hypoxic rats. In our conceptual definition of the two-module system, the producer module includes all the steps from $\mathrm{O}_{2}$ and substrates uptake to phosphorylation. So, the concept of direct effect of $\mathrm{O}_{2}$ quantified here embodies integrative properties of cellular steps as well as steps involved in the transfer of $\mathrm{O}_{2}$ from lung to mitochondria. The increase in hematocrit in HC rats (52 vs. 42\%) indicates improved $\mathrm{O}_{2}$ transport capacity; the lower Cox activity might indicate down-regulations in cell metabolism. Yet, these responses are not sufficient to assess in what extent the lower sensitivity of ATP production to $\mathrm{O}_{2}$ is due to adaptations in systemic $\mathrm{O}_{2}$ delivery [23] or due to cellular adaptations to chronic hypoxia [25]. A better understanding might come from in vivo assessments of muscle $\mathrm{PO}_{2}$ as achieved for instance by proton MR spectroscopy of deoxymyoglobin [26]. Unfortunately, due the low sensitivity of magnetic resonance, we are not aware of deoxymyoglobin assessments in small muscles of animals.

An interesting relationship to other ${ }^{31} \mathrm{P}$ MR approaches could be mentioned. As force is supposed to reflect the oxidative ATP rate (called $\mathrm{J}$ ) in our conditions and assuming 
(reasonably, in this largely aerobic exercise) usual linearity of $\mathrm{J}$ to $\mathrm{PCr}$, the location of the hypoxia point in $\mathrm{N}$ and $\mathrm{HC}$ rats in figure 3 correspond to a decreased $\Delta \mathrm{J} / \Delta \mathrm{PCr}$, which would correspond to a reduced rate constant (increased time constant) of PCr kinetics during onset or offset of exercise (recovery from stimulation). Such a decease in $\mathrm{PCr}$ recovery rate constant has been noted in a variety of experimental and pathological muscle-hypoxic states, and amounts to about $-50 \%$ in healthy humans breathing $10 \% \mathrm{O}_{2}$ [27]. This value could be compared to $-44 \%$, the direct effect of hypoxia on producer assessed here by regulation analysis in $\mathrm{N}$ rats. We are not aware of assessment of PCr recovery rate constants in $\mathrm{HC}$ animals breathing hypoxic mixtures. At last, it is worth noting that PCr recovery rate (or time) constants say nothing about the elasticity of ATP/PCr production and ATP/PCr consumption. Elasticities determine the regulation of ATP turnover in contracting muscle, are uniquely determined by MCA, and may thus be used to uncover the origin of the observed dysfunctions under hypoxia conditions [8].

In conclusion, the present study provides a quantitative approach of the effects of acute and long-term hypoxia on muscle energetics. In vivo assessments of elasticity coefficients and direct effect of $\mathrm{O}_{2}$ on ATP production illustrate how internal and external regulation of ATP turnover and contraction can be uncovered. We anticipate that the approach is useful to detect dysfunctions or improved functions at system levels [28] and brings new tools for integrative physiology [29]. 


\section{Elasticity Coefficients}

ATP/PCr-producer

$-1.6 \pm 1.4 \quad(20)$

$-1.7 \pm 0.8$

$\mathrm{ATP} / \mathrm{PCr}$-consumer

$0.8 \pm 0.6$

(28)

$1.1 \pm 0.7$

\section{Flux Control Coefficients}

ATP/PCr -producer

$34 \pm 23 \% \quad(20)$

$35 \pm 17 \%$

ATP/PCr -consumer

$66 \pm 23 \% \quad(20)$

$65 \pm 17 \%$

\section{Direct effect of acute hypoxia}

on $\mathrm{ATP} / \mathrm{PCr}$-Producer

Table 1. Coefficients determined by the control and regulation analyses

Values are mean \pm standard deviation. The number of experiments is indicated in parentheses.

See text for the definition of the calculated coefficients. $* P<0.05$ 


\section{Figure legends}

\section{Figure 1. The defined modular system and elasticities quantified by control and} regulation analyses. The energetic system of contraction in skeletal muscle in vivo was defined as two modules: ATP/PCr -producer and ATP/PCr -consumer. The consumer module embodies all the cellular ATPases that hydrolysis ATP. The producer module embodies all systemic and cellular steps that allow ATP production in active myocytes.

Figure 2. Typical recordings in a hypoxic conditioned (HC) rat. Recordings were obtained after the transitory period (not shown). Lowest peaks of PCr (front) indicate the period during which the $\mathrm{HC}$ rat inhaled $9 \% \mathrm{O}_{2}$; correspondingly contraction was low as indicated by pedal strokes due to plantar flexion. Highest peaks of PCr (back) were obtained during the lowelectrical stimulation period; correspondingly contraction was low. The 'reference' steady state is characterized by PCr-peaks of intermediate height and intermediate level of contraction. Elasticities were calculated from averaged pedal strokes and spectra. Pi, inorganic phosphate, NTP, nucleosides triphosphate.

Figure 3. Effects of modulations on PCr and contraction. Lines indicate effects of acute hypoxia and lowering electrical stimulation on steady values of $\mathrm{PCr}$ and contraction in $\mathrm{N}$ rats (squares) and $\mathrm{HC}$ rats (circles). Therefore, slopes provide a rough illustration of elasticities. Slopes look similar in $\mathrm{N}$ and $\mathrm{HC}$ rats, as confirmed by the actual calculation of elasticity coefficients (table 1) thanks to control analysis. Arrows show the more marked effect of acute hypoxia in $\mathrm{N}$ rats, studied here by tools of regulation analysis. 


\section{REFERENCES}

1 Haseler, L. J., Richardson, R. S., Videen, J. S. and Hogan, M. C. (1998) Phosphocreatine hydrolysis during submaximal exercise: the effect of FIO2. J Appl Physiol 85, 1457-1463

2 Hogan, M. C., Arthur, P. G., Bebout, D. E., Hochachka, P. W. and Wagner, P. D. (1992) Role of O2 in regulating tissue respiration in dog muscle working in situ. J Appl Physiol 73, 728-736

3 Bylund-Fellenius, A. C., Walker, P. M., Elander, A., Holm, S., Holm, J. and Schersten, T. (1981) Energy metabolism in relation to oxygen partial pressure in human skeletal muscle during exercise. Biochem J 200, 247-255

4 Idstrom, J. P., Subramanian, V. H., Chance, B., Schersten, T. and Bylund-Fellenius, A. C. (1986) Energy metabolism in relation to oxygen supply in contracting rat skeletal muscle. Fed Proc 45, 2937-2941

5 Allen, P. S., Matheson, G. O., Zhu, G., Gheorgiu, D., Dunlop, R. S., Falconer, T., Stanley, C. and Hochachka, P. W. (1997) Simultaneous 31P MRS of the soleus and gastrocnemius in Sherpas during graded calf muscle exercise. Am J Physiol 273, R999-1007

6 Matheson, G. O., Allen, P. S., Ellinger, D. C., Hanstock, C. C., Gheorghiu, D., McKenzie, D. C., Stanley, C., Parkhouse, W. S. and Hochachka, P. W. (1991) Skeletal muscle metabolism and work capacity: a 31P-NMR study of Andean natives and lowlanders. J Appl Physiol 70, 1963-1976

7 Arsac, L. M., Beuste, C., Miraux, S., Deschodt-Arsac, V., Thiaudiere, E., Franconi, J. M. and Diolez, P. H. (2008) In vivo modular control analysis of energy metabolism in contracting skeletal muscle. Biochem J 414, 391-397

8 Calmettes, G., Deschodt-Arsac, V., Thiaudiere, E., Muller, B. and Diolez, P. (2008) Modular control analysis of effects of chronic hypoxia on mouse heart. Am J Physiol Regul Integr Comp Physiol 295, R1891-1897

9 Diolez, P., Deschodt-Arsac, V., Raffard, G., Simon, C., Santos, P. D., Thiaudiere, E., Arsac, L. and Franconi, J. M. (2007) Modular regulation analysis of heart contraction: application to in situ demonstration of a direct mitochondrial activation by calcium in beating heart. Am J Physiol Regul Integr Comp Physiol 293, R13-19

10 Korzeniewski, B., Deschodt-Arsac, V., Calmettes, G., Franconi, J. M. and Diolez, P. (2008) Physiological heart activation by adrenaline involves parallel activation of ATP usage and supply. Biochem J 413, 343-347

11 Ainscow, E. K. and Brand, M. D. (1999) Quantifying elasticity analysis: how external effectors cause changes to metabolic systems. Biosystems 49, 151-159

12 Brand, M. D. (1997) Regulation analysis of energy metabolism. J Exp Biol 200, 193202

13 Brand, M. D. (1998) Top-down elasticity analysis and its application to energy metabolism in isolated mitochondria and intact cells. Mol Cell Biochem 184, 13-20

14 Brand, M. D. and Curtis, R. K. (2002) Simplifying metabolic complexity. Biochem Soc Trans 30, 25-30

15 Fell, D. A. (1992) Metabolic control analysis: a survey of its theoretical and experimental development. Biochem J 286 ( Pt 2), 313-330

16 Jeneson, J. A. (2002) Regulation analysis of contractile ATPase flux in skeletal muscle. Mol Biol Rep 29, 167-170 
17 Jeneson, J. A., Westerhoff, H. V. and Kushmerick, M. J. (2000) A metabolic control analysis of kinetic controls in ATP free energy metabolism in contracting skeletal muscle. Am J Physiol Cell Physiol 279, C813-832

18 Arthur, P. G., Hogan, M. C., Bebout, D. E., Wagner, P. D. and Hochachka, P. W. (1992) Modeling the effects of hypoxia on ATP turnover in exercising muscle. J Appl Physiol 73, 737-742

19 Nioka, S., Argov, Z., Dobson, G. P., Forster, R. E., Subramanian, H. V., Veech, R. L. and Chance, B. (1992) Substrate regulation of mitochondrial oxidative phosphorylation in hypercapnic rabbit muscle. J Appl Physiol 72, 521-528

20 Wu, F., Jeneson, J. A. and Beard, D. A. (2007) Oxidative ATP synthesis in skeletal muscle is controlled by substrate feedback. Am J Physiol Cell Physiol 292, C115-124

21 Connett, R. J. (1988) Analysis of metabolic control: new insights using scaled creatine kinase model. Am J Physiol 254, R949-959

22 Meyer, R. A. (1988) A linear model of muscle respiration explains monoexponential phosphocreatine changes. Am J Physiol 254, C548-553

23 Wolfel, E. E., Groves, B. M., Brooks, G. A., Butterfield, G. E., Mazzeo, R. S., Moore, L. G., Sutton, J. R., Bender, P. R., Dahms, T. E., McCullough, R. E. and et al. (1991) Oxygen transport during steady-state submaximal exercise in chronic hypoxia. J Appl Physiol 70, 1129-1136

24 Hogan, M. C., Kurdak, S. S. and Arthur, P. G. (1996) Effect of gradual reduction in O2 delivery on intracellular homeostasis in contracting skeletal muscle. J Appl Physiol 80, 1313-1321

25 De Palma, S., Ripamonti, M., Vigano, A., Moriggi, M., Capitanio, D., Samaja, M., Milano, G., Cerretelli, P., Wait, R. and Gelfi, C. (2007) Metabolic modulation induced by chronic hypoxia in rats using a comparative proteomic analysis of skeletal muscle tissue. J Proteome Res 6, 1974-1984

26 Richardson, R. S., Noyszewski, E. A., Kendrick, K. F., Leigh, J. S. and Wagner, P. D. (1995) Myoglobin O2 desaturation during exercise. Evidence of limited O2 transport. J Clin Invest 96, 1916-1926

27 Haseler, L. J., Lin, A. P. and Richardson, R. S. (2004) Skeletal muscle oxidative metabolism in sedentary humans: 31P-MRS assessment of O2 supply and demand limitations. J Appl Physiol 97, 1077-1081

28 Noble, D. (2003) The future: putting Humpty-Dumpty together again. Biochem Soc Trans 31, 156-158

29 Strange, K. (2005) The end of "naive reductionism": rise of systems biology or renaissance of physiology? Am J Physiol Cell Physiol 288, C968-974 


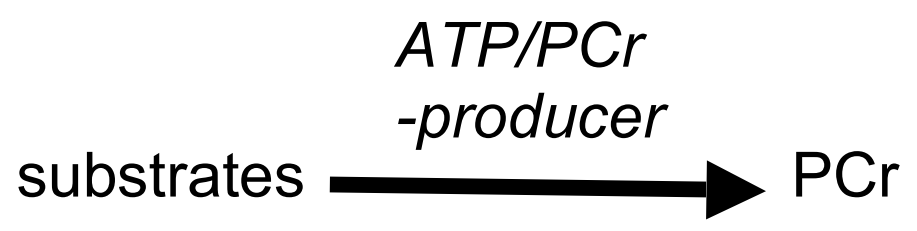

figure 1 


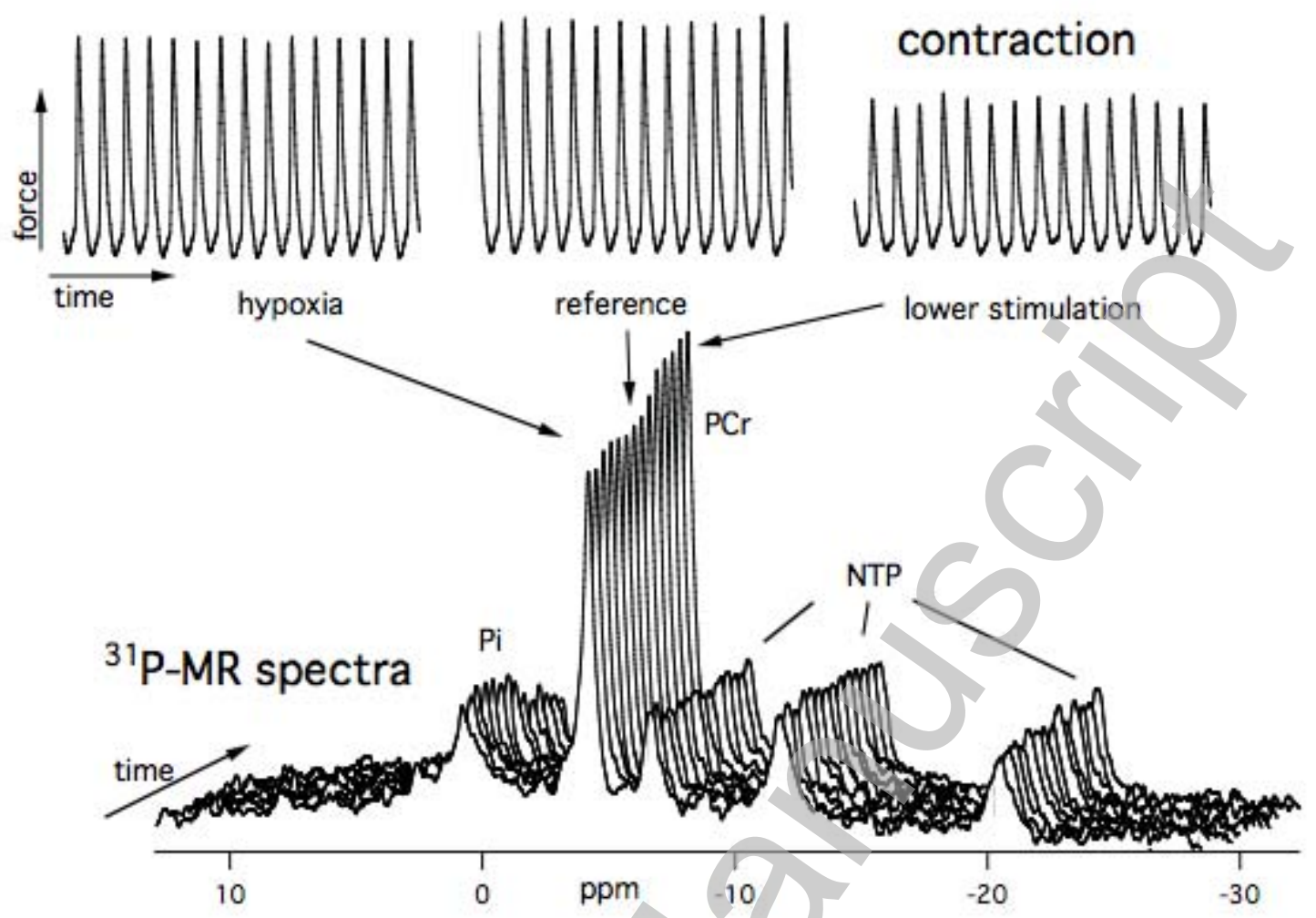

figure 2 


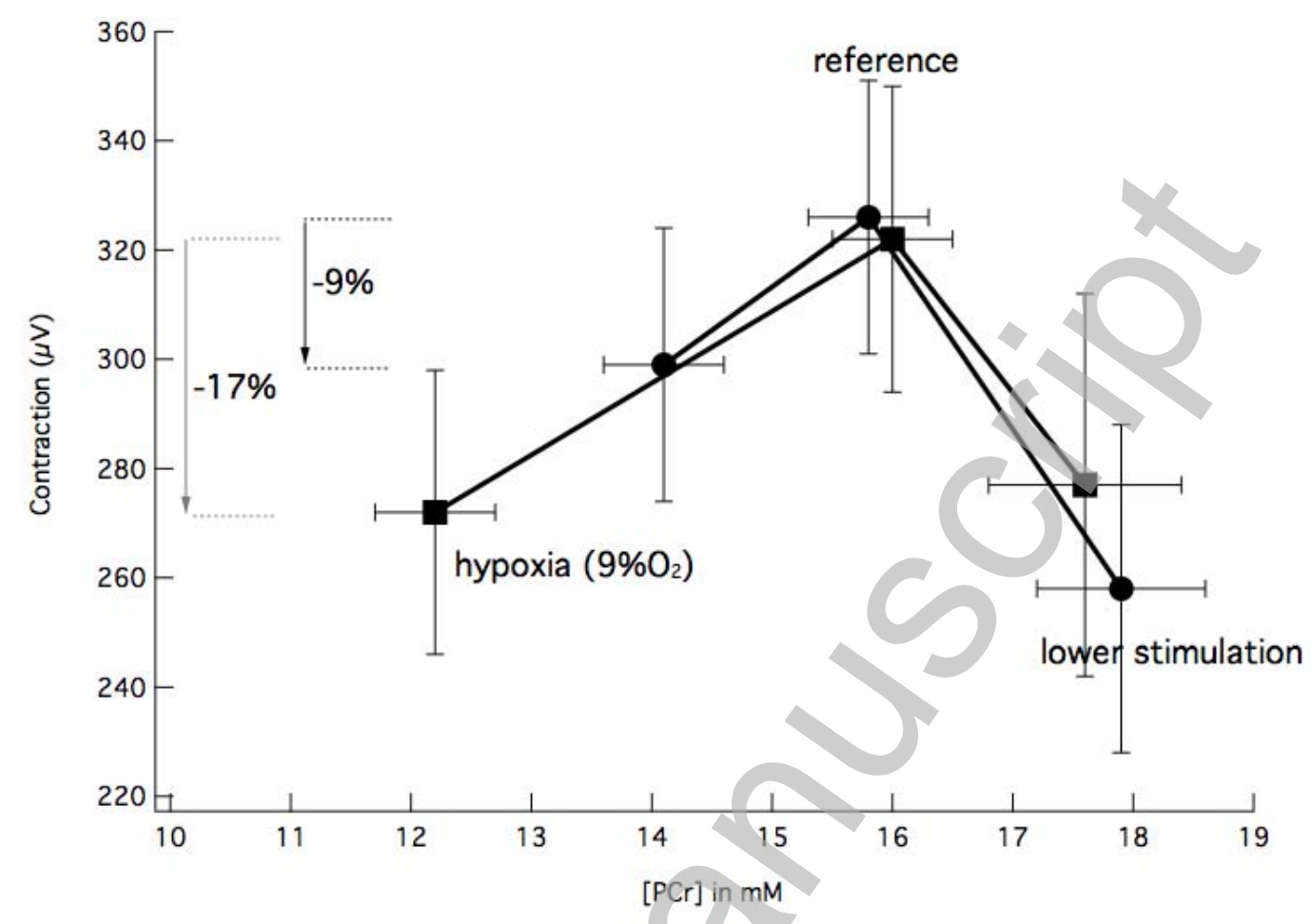

figure 3 